SPECIAL REPORT

\title{
CHILD SEXUAL ABUSE; COMPARISON OF KNOWLEDGE AND ALERTNESS AMONG GENDERS
}

\begin{abstract}
Child sexual abuse (CSA) is a significant health problem with potential long-term consequences for victims, therefore knowledge regarding sexual abuse is essential for children. Educating children about body parts, good and bad touch develops positive body image and self-confidence. Informed and confident children are more likely to prevent themselves from abuse and reach out for the help. This quantitative descriptive survey based study evaluates and compares the knowledge and alertness against CSA among children of both genders. 100 participants of age bracket 6 to 12 years old both genders from different areas of Karachi were selected through simple random sampling technique. Informed consent was taken from the parents of the participants. The survey of 20-25 minutes was done in a course of 2 weeks in a semi structures setting. Each question was explained to children taken in group of 5 , in simple and comprehensible Urdu language. Assessment was done using The Child Knowledge of Abuse Questionnaire - Rev II (CKAQ-REV II). Result indicates negligible difference of knowledge and skills about CSA between girls and boys. More than average of the children in the study have marked 'False' and 'I don't know' on the application based questions that depict poor practical skills. Mean score of girls and boys on CKAQ-REV II is 16 and 17 respectively that shows children of Pakistani society are at high risk of CSA. Similar study can be performed to find the correlation of knowledge and awareness of CSA between children and their parents.
\end{abstract}

\section{KEYWORDS}

Child sexual abuse, knowledge, abuse, questionnaire, survey, Pakistani society, sexual trauma

\section{Mahnoor Anwar}

Occupational Therapist

Institute of Physical Medicine \& Rehabilitation

Dow University of Health Sciences

mah.anwar31@gmail.com

\section{Fatima Khan}

Senior Lecturer

Ziauddin College of Rehabilitation Sciences

Ziauddin University

Fatima@zu.edu.pk

[Anwar M, Khan F. Child sexual abuse; comparison of knowledge and alertness among genders. Pak. j. rehabil. 2018;7(1):48-56] 


\section{INTRODUCTION}

Child sexual abuse (CSA) is a serious global health issue with lifelong consequences for the survivors, therefore knowledge regarding sexual abuse is essential for children. CSA occurs when a child is forced to take part in sexual activities. It can vary in forms like verbal abuse, online abuse and much more. CSA is generally divided into two broad categories i.e. contact abuse and non-contact abuse. In contrast to non-contact abuse, contact abuse involves activities in which the abuser makes physical contact with the child; it may include penetration while the non-contact abuse includes non-touching activities such as pornography'. CSA is a global health issue of great magnitude affecting children of all ages, genders, races, ethnicity and socioeconomic classes. The offender is often an adult but can be another child too. The vast majority of sexual offenders are someone the child and the family already know and trust. The knowledge of sexual abuse is demand of present time while alertness is essential.

CSA is a type of trauma that instills guilt in the victims. In childhood sexual abuse, the victim is too young to take appropriate action and seek out for help. If it is not addressed on time, it can lead to lifelong psychological problems. The offender is often the close friend or associate to the family which further creates hindrance to report CSA. Present quantitative study aims to compare and evaluate child's knowledge and alertness in boys and girls against child sexual abuse.

Recently, there has been increased attention to CSA as a global issue for which solutions must be devised in order to protect children from further harm and prevent others from becoming victims. Although CSA is a widespread phenomenon, it remains difficult to collect reliable prevalence data. A meta-analysis conducted by Stoltenberg et al. looking at worldwide data revealed that the prevalence of self-reported childhood sexual abuse cases was $11.8 \%$, with $18 \%$ prevalence for girls and $7.6 \%$ for boys between 1980 and $2008^{2}$. In another study approximately 5.6 percent of adolescents 15-19 years of age reported that they felt pressured the first time they had sex, with adolescent girls more likely to feel pressure than boys (9.7 percent and 2.5 percent respectively) ${ }^{3}$. Another study shows self-reported CSA cases are more common in girls $(133 / 1000)$ than boys (41/1000) in $\mathrm{Asia}^{4}$.

In Pakistan an organization named Sahil works on child protection while keeping a keen focus on child sexual abuse, the organization publish yearly report by gathering data from different sources like newspaper (national and regional), media reports, police reports and unregistered parent reports. Latest finding of year 2017 reveals that in a day more than 9 children have been sexually abuse in the country. The total 3445 child abuse cases were reported in newspapers from all four provinces in the year $2017^{5}$. The present number of cases is only the small portion of total cases as many cases remain unreported, actual number of CSA in Pakistan can be assumed to be much higher.

Child Sexual Abuse (CSA) is one of the biggest social and health problems around the globe with long term adverse psychological and social consequences. CSA link to lifetime psychopathologies have been demonstrated by many clinicians and researchers ${ }^{6}$. Despite overwhelming evidences of negative psychological consequences of CSA, study shows that impact of CSA is variable and many victims do not exhibit clinical level of symptoms. It is secondary to the fact that CSA has diverse characteristics that can cause wide range of outcomes. The other reasons may include asymptomatic survivors that have developed avoidant and other coping styles and insufficient severity of abuse. The asymptomatic survivors are more resilient and can deal with distress more effectively than symptomatic survivors ${ }^{6-8}$.

According to one study, CSA and other child maltreatments are found to be one of the major risk factor to develop Non suicidal self-harm injuries later in adulthood'. Many mental health problems other than post-traumatic stress disorder and disturbance in behavior are linked to CSA. Symptoms of Major Depressive disorders are found in victimized children. Sexually abused children are more likely to exhibit sexualized behaviors than their non-abused counterparts. These children during their teenage period are more likely to get involve in substance abuse and self-harm behaviors ${ }^{10-11}$.

CSA is a subject of great annoyance and discomposure across all the cultures despite it is highly widespread across the world. The effects of CSA may not always show initially but carries serious threat to their well-being. Despite this fact, it is one of the most unaddressed subjects across all the cultures. It is true to the fact that too much social taboos are attached to it. Victim fears to report the abuse out of fear of rejection, guilt and shame. Angie C. Kennedy found that CSA, intimate partner violence, Sexual Assault survivors experienced stigma including victim blaming messages from their disclosure. Such reaction causes survivors to internalize shame and self-blame ${ }^{12}$.

According to one of the estimation, $15-20 \%$ of girls and boys from all socioeconomic groups in Pakistan are exposed to sexual harassment and abuse before reaching 18 years (The Bitter Truth-Rozan), with many cases going unreported or undetected, it can be assumed that the real statistics will be much higher ${ }^{13}$. One of the reasons of high prevalence of CSA is the ignorance. A large number of children around the globe are not given education 
about sexual abuse. Many adults do not have knowledge and skills to identify changing behaviors in their children stemming from CSA ${ }^{11}$.

According to experts, if children are given education and taught skills to respond to bad touch, the CSA incidence can be controlled. Assertive and well-informed children are more likely to protect themselves from abuse ${ }^{11}$.

The study was conducted in China, Beijing by Yichen Jin, Jingqi Chen \& Buyi Yu (2016) to examine level of knowledge against prevention of child sexual abuse. This study was conducted on school aged children from grade 1 to 5, 559 participants from one primary school took part in the study. The respondents were asked to fill questionnaire which was about knowledge and skills related to CSA prevention. The result of this study revealed the truth that majority of the children lacked knowledge and skills and thus education programs related to CSA should be introduced in schools particularly in the elementary classes ${ }^{14}$

Child sexual abuse (CSA) is a significant health problem with potential long-term consequences for victims, therefore knowledge regarding sexual abuse is essential for children. Equipping children with knowledge of CSA is very essentially as they cannot be always protected and guarded by their parents. Well informed children will respond assertively to such happening and will reach out for the help. This is a quantitative study to evaluate and compares the knowledge and alertness against child sexual abuse among children of both genders.

\section{METHODOLOGY}

The present study is descriptive survey based research. The sample of 100 children of both genders in age bracket 6 yrs to 12 yrs. old were selected through Simple Random technique from different areas of Karachi. These children were not provided with any formal training about Child Sexual Abuse prior to the study. The survey was done in a course of 2 weeks. Data collection tool used was Child Knowledge of Abuse Questionnaire - Rev II. The Children were divided into 2 groups according to their gender. Each question was explained to them in simple and comprehensible Urdu language followed by 20 to 25 minutes to complete the survey form. The children with Iow IQ and those who have got formal training against Child Sexual abuse in past 6 months in schools, summer camps or any other organization were excluded from the study. Data analysis was done by SPSS version 20 , t-test applied.

Parents of the selected children were briefed about the research objectives and method. They were explained about the Questionnaire. Prior informed consent was taken from the parents of children observed under the study.

\section{RESULT}

Results of this study showed that $56 \%$ of the females $(n=50)$ and $52 \%$ of males responded true in item-10 of CKAQ-REV II. 62\% females and 52\% males answered true in response to item-12, similarly for item-22 $61 \%$ and $56 \%$ female and male participants responded true while $41 \%$ and $64 \%$ responded true in item-31.For item-32, 32\% females and $30 \%$ males marked true. Overall, the obtained responses varied according to gender perceptions (Figure $1-2)$.

\section{DISCUSSION}

Both quantitative and qualitative item analysis convincingly revealed that there is no significant difference in the knowledge of CSA concepts between genders as measured by the CKAQ-REV II. As the average difference between scores of females and males on CKAQ-REV $\|$ is small so it is negligible. Thus we conclude that there is no significance difference of knowledge between girls and boys about child sexual abuse. This is true to the fact that in our society the children of either sex do not get proper education and awareness of child sexual abuse neither in school or home.

Child sexual abuse is disturbing and intimidating topic for parents and other care givers to address with children. Often caregivers fear that discussing the topic may frighten children and take away their innocence from them. Furthermore, there is still a great deal of stigma and shame associated with violence and abuse in our society that stops people from addressing issues and educating their children. This is the reason that child sexual abuse cases are increasing in our society day by day and yet our children are not prepare for it. By looking at the table no. 1 we can easily infer that girls have $48 \%$ of knowledge of child sexual abuse and boys have $51 \%$ of knowledge which falls in the alarming level.

Figure 1-2 shows that more than $50 \%$ of the children have marked application based questions true or I don't know. We can infer from the reading (Figure 1-2) that children lack skills to execute their knowledge in real life situations. Majority of the children cannot discriminate between safe and unsafe situations and act accordingly. Thus these children are at high risk of abuse.

The results of the present study closely resembles to the study conducted by Wenjing Zhang and his fellows in Beijing, China. The researchers interviewed 
136 children to examine the level of knowledge and skills related to CSA. The result showed lack of knowledge and skills about CSA in Chinese children. They found that less than half of the total children can discriminate stranger versus non stranger, only $16 \%$ of the children reported that bad touch should be reported, less than $30 \%$ of the children were aware about using verbal responses to refuse inappropri- ate touches which shows lack of knowledge and awareness. The parents of these children were also interviewed. Qualitative analysis showed that parents too lack knowledge and skills to train their children about CSA. In the same study less than half of the total parents discussed about good and bad touch and safe and unsafe places with their children ${ }^{15}$.

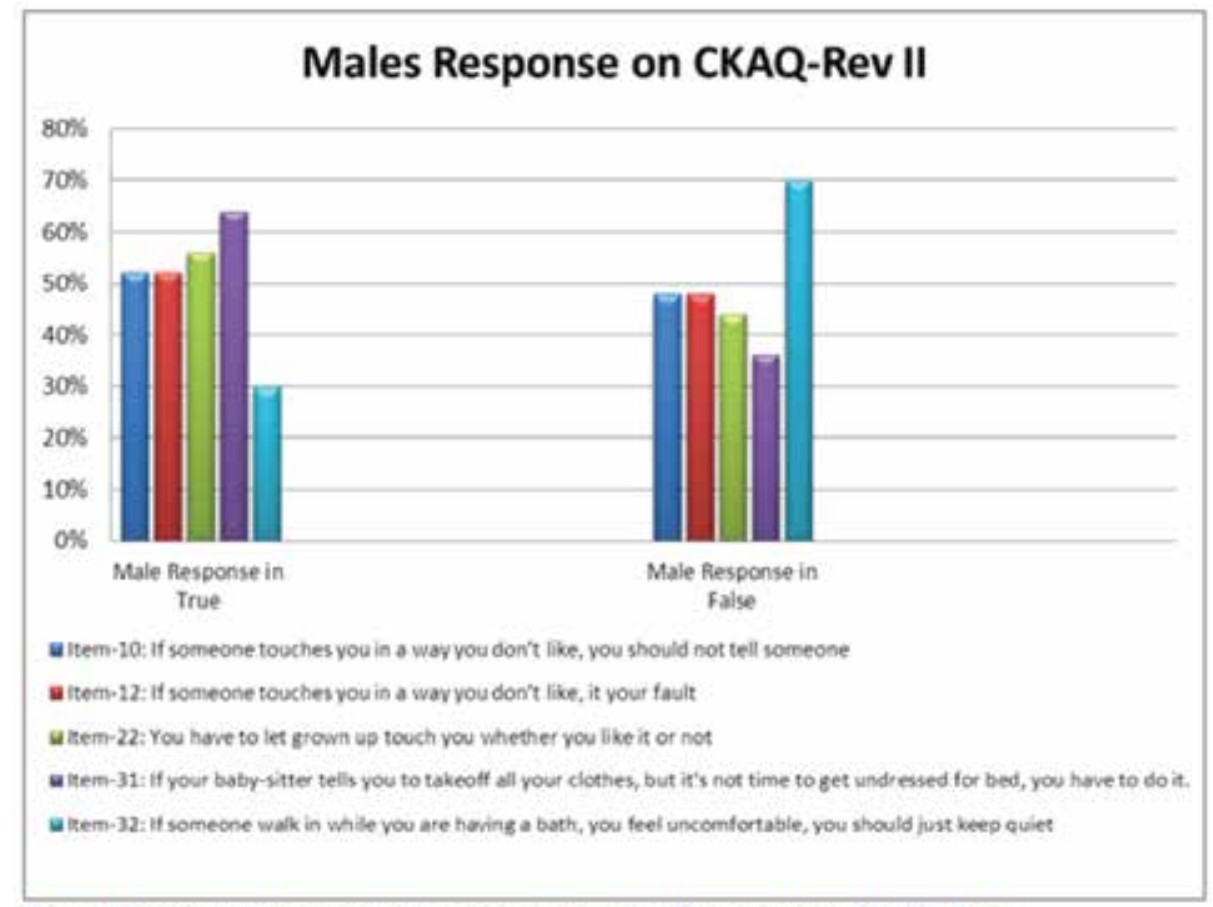

Figure 1 showing the male participants response on different items of CKAQ-Rev II

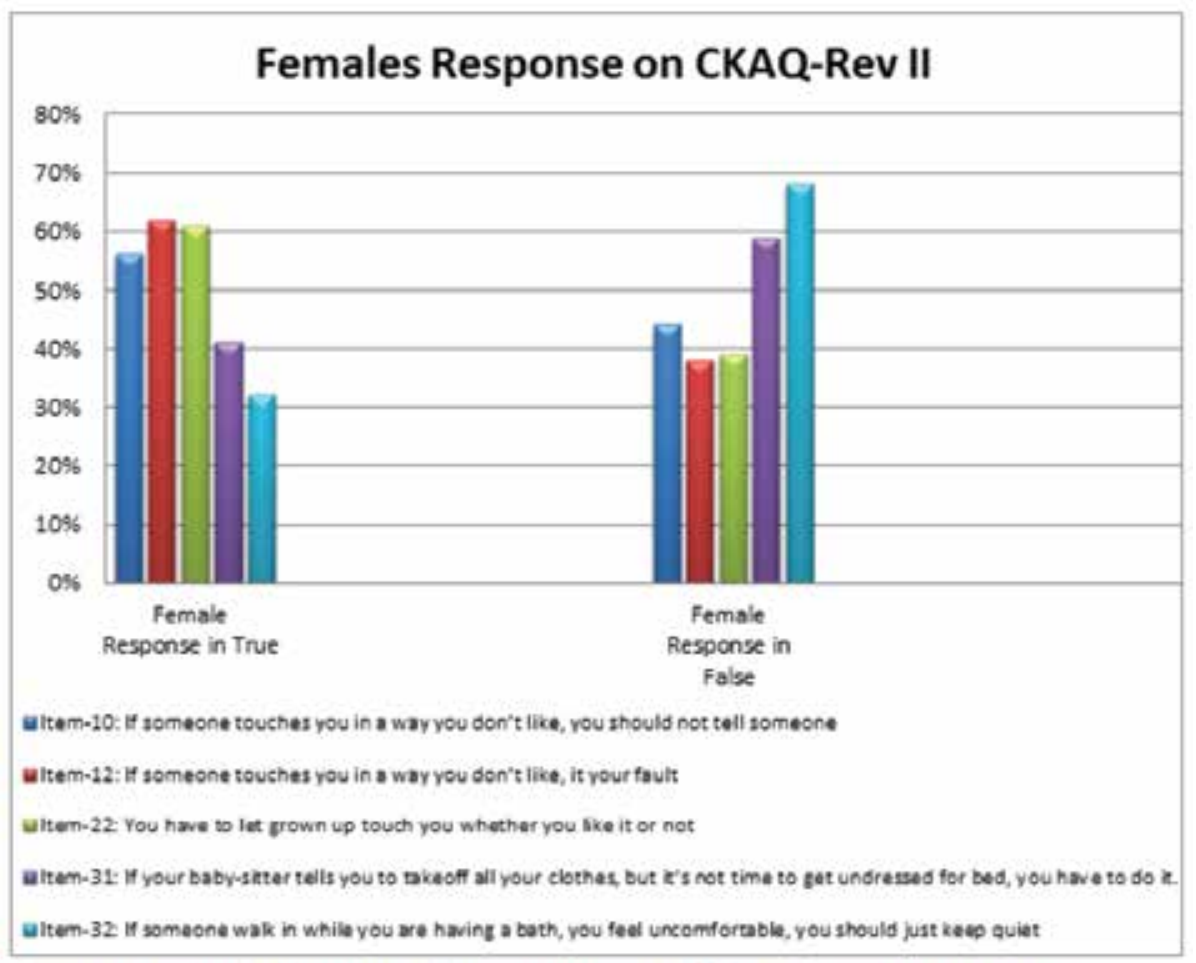

Figure 2 showing the female participants response on different items of CKAQ-Rev II 


\section{Limitation and Recommendations}

The questionnaire used in the study was very complicated for children of age group 6-12 years old to understand no subscale was given which made it difficult for researcher to determine knowledge and alertness individually further determinants like basic knowledge of private body parts, good touch and bad touch questions was not categorize separately, if it was categorize in sections than interpretation and scoring would have been more specific. This questionnaire can be modified by adding below mentioned points.

- Items can be divided into sub headings of knowledge, application and understanding of child sexual abuse.

- Questions to judge knowledge about private body parts, private and public places can be added.

- Question can be made more comprehensible and simple for children to understand.

- Question to analyze child skills of incorporating knowledge into practical life situations can be added.

This study provides prospect for many future studies on wider population. As the parents and teachers are the primary informants to children, their attitudes, awareness knowledge and myths can be studied and correlated with the knowledge of the children. School curriculum can be explored too to examine the contribution of school in preparing the children. This will provide a way to work on the gaps and control the CSA incidents.

Children with physical and mental disabilities are more vulnerable to abuse than their counterparts as they lack the skills and understanding to respond effectively 16. Further studies can be done on this population to assess and evaluate the parental involvement in educating their disabled children about CSA.

\section{CONCLUSION}

CSA occurs when child is forced into sexual activity. It has long-lasting devastating effects on the victim's wellbeing. Its prevalence is increasing day by day around the globe affecting both genders irrespective of socioeconomic background. Despite of its increasing prevalence, it is least addressed. Children of Pakistani society are at high risk of CSA as they lack knowledge and skills.

\section{ACKNOWLEDGEMENT}

We would like to show our sincere gratitude to Dr. Anjum Ara, Lecturer, Department of Clinical Psychology University of Karachi for mentoring us throughout the process.

\section{REFRENCES}

[1] Krishnan S, Syahirah N, Syahirah N, Amira N. Study on Child Sexual Abuse. Human resource management research. 2017;7(1): 38-42

[2] Stoltenborgh $M$, Van ljzendoorn $M H$, Euser EM, Bakermans-Kranenburg MJ. A global perspective on child sexual abuse: meta-analysis of prevalence around the world. Child Maltreat. $2011 ; 16(2): 79-101$

[3] Rafael C, Karin AR, Yolanda G. Adolescent Sexual and Reproductive Health in El Salvador. Health, nutrition, and population (HNP) discussion paper. 2015. 1 https://openknowledge.worldbank.org/bitstream/handle/10986/23687/AdolescentOsexOealthOinOEIOS alvador.pdf? sequence $=1$

[4] Collin-Vézina D, Daigneault I, Hébert M. Lessons learned from child sexual abuse research: prevalence, outcomes, and preventive strategies. Child Adolesc. Ment. Health. 2013;7(1):22.

[5] A compilation of statistics on child sexual abuse of reported cases in Pakistan. Sahil. org. March 2018. Available from: http://sahil.org/cruel-numbers/

[6] Cutajar MC, Mullen PE, Ogloff JR, Thomas SD, Wells DL, Spataro J. Psychopathology in a large cohort of sexually abused children followed up to 43 years. Child abuse \& neglect. 2010;34(11):813-22

[7] Geffner R, Franey KC, Falconer R. Adult sexual offenders: Current Issues and Future Directions. J Child Sex Abus. 2003;12(3-4):1-16

[8] Cook A, Spinazzola J, Ford J, Lanktree C, Blaustein M, Cloitre M et al. Complex Trauma in Children and Adolescents. Psychiatr Ann. 2005; 35(5):390-398.

[9] Chartrand H, Bhaskaran J, Sareen J, Katz L, Bolton J. Correlates of Nonsuicidal Self-Injury and Suicide Attempts among Tertiary Care, Emergency Department Patients. Can J Psychiatry. 2015;60(6):276-283.

[10] Collin-Vézina D, Daigneault I, Hébert M. Lessons learned from child sexual abuse research: Prevalence, outcomes, and preventive strategies. Child Adolesc Psychiatry Ment Health. 2013;7(1):22.

[11] Kmett Danielson C, Macdonald A, Amstadter $A B$, Hanson R, de Arellano MA, Saunders BE, Kilpatrick DG. Risky behaviors and depression in conjunction with-or in the absence of-lifetime history of PTSD among sexually abused adolescents. Child Maltreat. 2010;15(1):101-7.

[12] Kennedy A, Prock K. "I Still Feel Like I Am Not Normal": A Review of the Role of Stigma and Stigmatization Among Female Survivors of Child Sexual Abuse, Sexual Assault, and Intimate Partner Violence. Trauma Violence Abuse. 2016;19(5):512-527.

[13] Hadi S. empowering your children and preventing sexual abuse. Aahung.org. April 2017. Available from: https://www.aahung.org/upload/re- 
sources/csa_english.pdf

[14] Jin Y, Chen J, YU B. Knowledge and skills of sexual abuse prevention: a study on school-aged children in Beijing, China. J Child Sex Abus 2016;25(6):686-96

[15] Zhang W, Chen J, Feng Y, Li J, Zhao X, Luo X. Young children's knowledge and skills related to sexual abuse prevention: A pilot study in Beijing,

China. Child Abuse \& Neglect. 2013; 37(9):623-630.

[16] Pulla V, Tarar M, Ali M. Child Protection System and Challenges in Pakistan. Space and Culture, India. 2018;5(3):54. 